\title{
Auditory and visual control over chained operants
}

RICHARD GILBERT AND MAURICE MOORE

ABERDEEN UNIVERSITY, SCOTLAND

Hooded rats trained to emit a simple two-operant chain under auditory control were less affected by omission of the exteroceptive stimulus correlated with the second component of the chain than were hooded rats trained to emit a similar cha in under the control of a local or distant visual source. This difference was unrelated to the required order of emission of the two operants.

Gilbert (1966a) trained rats to emit a simple fouroperant chain which was controlled at first mainly by various auditory stimuli as consequences and cues for the individual operants, and reported that the auditory stimuli no longer controlled the components of the chain after extensive practice. In a further study, Gilbert (1966b) found a marked difference in persistence of control by auditory and visual stimuli over the components of a simple six-operant chain. In the second study, auditory stimuli were occasions for pulling one or another of two trapezes, and visual stimuli were occasions for pressing one or another of four levers. The present study was carried out to examine the possible effect of this response difference, to compare the persistence of control by local and distant light sources, and to provide the possibility of a systematic replication of the difference between the persistence of auditory and visual control.

\section{Method}

Ten naive, male black-hooded rats were used. They were aged three months at the beginning of a onemonth deprivation program which stabilized body weights at $80 \pm 4 \%$ of free-feeding weights at three months of age.

Two Foringer Type 1107 test chambers were used, each with a $1107 \mathrm{~L}$ response lever in a side position and a $1107 \mathrm{CL}$ chain lever (trapeze) mounted centrally $7.5 \mathrm{~cm}$ from the instrument panel. A $1134 \mathrm{~B}$ stimulus light was set in a side panel as close to the rat's end of the response lever as possible. A $1107 \mathrm{HL}$ house light was mounted centrally near the top of the instrument panel. An additional $2.2 \mathrm{~W}$ lamp providing dim illumination of the chamber and a small loudspeaker were both mounted behind the instrument panel, together with a 1284 pellet dispenser which delivered one $45 \mathrm{mg}$ Noyes pellet on command. The chamber was contained in a sound-proofed box which was in a sound-proofed cubicle in a room containing the electromagnetic controlling circuitry.

After deprivation, two rats were allocated to each of five conditions specified in terms of the order of operants required (lever $\rightarrow$ trapeze, or trapeze $\rightarrow$ lever) and the stimulus providing the occasion for emission of the second operant (lever light, house light, or approximately 400 cycle, medium intensity tone from a Foringer 1166-4 multiple stimulus panel). The "lever $\rightarrow$ trapeze/lever light" condition was not used. Each rat was trained first to emit the operant which was to be the second component of the required two-operant chain. Second, each rat was trained to emit this operant in the presence of the appropriate stimulus, and not in its absence, using a temporal fading procedure applied so as to minimize occurrence of inappropriate responses. All appropriate responses were reinforced. When emission of the operant during a $20 \mathrm{sec}$ absence of the stimulus was negligible, and the operant was emitted within $2 \mathrm{sec}$ of onset of the stimulus, the other operandum was introduced. The stimulus, and thus the availability of reinforcement, could now be produced by successive approximations to, and eventually emission of, the other operant. (It seemed that less shaping of the new operant was required when it produced an auditory stimulus than when it produced a visual stimulus.) The stimulus was removed with reinforcement delivery, reinstating conditions for appropriate emission of the first operant of the chain. Inappropriate responding (errors), i.e., the second operant in the absence of the stimulus or the first operant in the presence of the stimulus, had no consequence.

Each rat was then run for eight daily sessions of 120 trials, each trial terminating in reinforcement delivery. Throughout six of these sessions the contingencies were the same as at the end of pretraining. Sessions 2 and 7 were also run under the same conditions except that following the 20th, 60th, and 100th reinforcements the stimulus was not available for 20 trials, although the other contingencies remained the same during these trials.

\section{Results}

Errors at each stage of the chain were cumulated by blocks of 20 trials, and the time taken to complete each block was recorded. Errors within $0.5 \mathrm{sec}$ of an appropriate response were ignored, to avoid the occasional contamination of an error count by subsequent responses in short bursts which began with an appropriate response. Of these three measures, only errors in the second stage of the chain were stable within conditions and varied consistently between some conditions. Error counts are represented in Fig. 1, which suggests that omission of the tone had no effect upon behavior, whereas omission of either of the light stimuli produced a considerable increase in inappropriate responding during the second stage in all cases. Neither the locality of the light, nor the required order of omission of the two operants, 


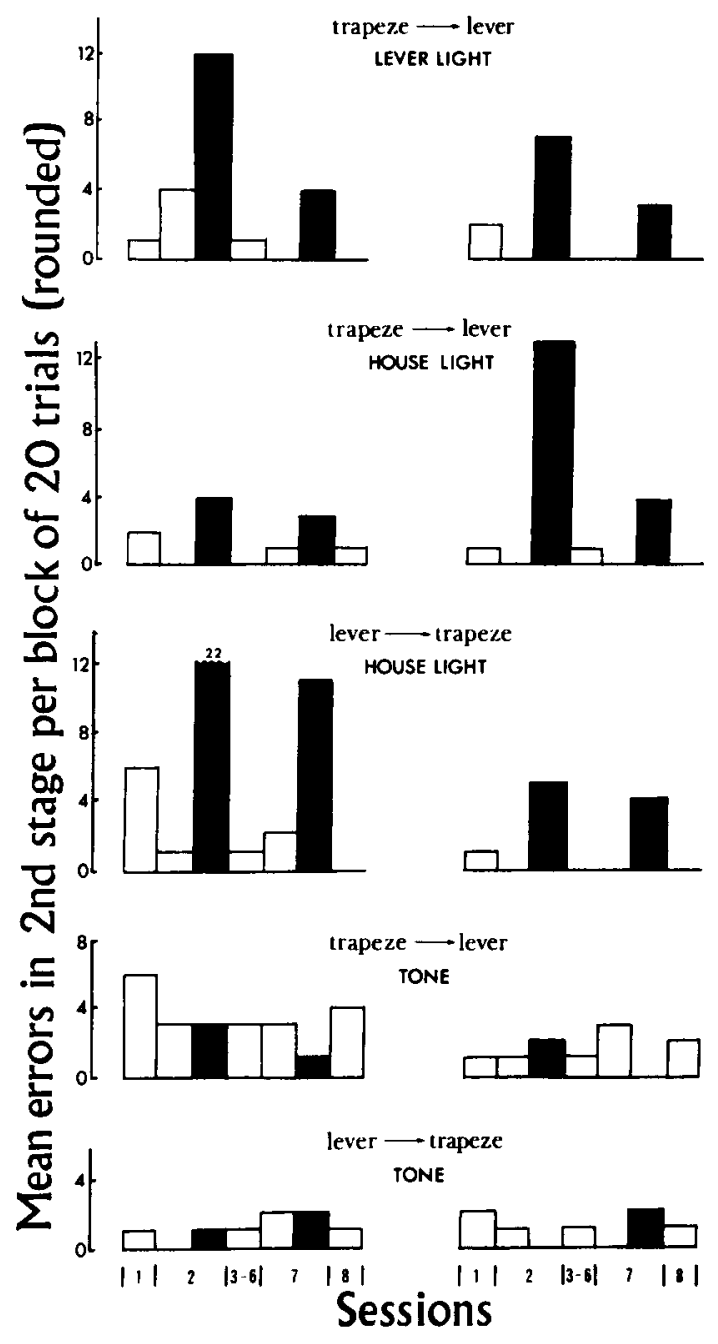

Fig. 1. Mean errors per block of 20 trials, to the nearest whole number, in second stage of chain, arranged by sessions or parts of sessions, for 10 rats each under one of tive conditions. Solid bars show error counts when stimulus was omitted. An error is specified more closely in the text.

seems from these results to be a relevant variable. A "practice" effect may be noticed, in that visual control was less during the second set of omissions than during the first set.

\section{Discussion}

These results are in accord with the Gilbert (1966b) study. However, as in that study, the first test of exteroceptive stimulus control was given too late in training to pick up control by the auditory stimuli. If it is to be assumed that a simple chain initially controlled by auditory stimuli soon comes under the control of another source (e.g., proprioceptive or visual) then both the point at which this occurs and the new source of control should be demonstrated.

These results may be contrasted with those of Field \& Boren (1963) who found that an auditory added clock was more effective in controlling behavior under an adjusting avoidance schedule than a visual added clock, in that under the auditory clock the rats stayed closer in time to the shock than under the visual clock. Apart from strain and stimulus differences between that study and the present one, the discrepancy may also be related to the type of behavior required under the two schedules. In the Field \& Boren study, efficient behavior depended upon both engaging in (consistent?) behavior other than lever pressing, and approaching the lever when a particular clock indication occurred. The animals may have had to remain orientated towards the lights above the lever when under control of the visual clock, and thus may have been more likely to press the lever. Under the conditions of the present study, such orientating behavior is presumably efficient. Hence, possibly, the greater dependence upon the visual source which, even when relatively distant, may have provided sufficient illumination of the lever to enhance orientation.

\section{References}

Field, G. E., \& Boren, J. J. An adjusting avoidance procedure with multiple auditory and visual warning stimuli. J. exp. Anal. Behav., 1963, 6, 537-543.

Gilbert, R. M. Manipulation and extinction of operant chains. In Proceedings of the XVIIIth International Congress of Psychology, Moscow, 1966a, 1, 140-141. (Abstract)

Gilbert, R.M. Some studies of chained operants. Unpublished Ph.D. thesis, Queen's University, Belfast, Northern Ireland, 1966b. 